\title{
e-/e+ ACCELERATING STRUCTURE WITH CYCLIC VARIATION OF AZIMUTH ASYMMETRY
}

\author{
Anatoly Krasnykh, Stanford Linear Accelerator Center, Menlo Park, California 94025
}

\begin{abstract}
A classical electron/positron accelerating structure is a disk-loaded cylindrical waveguide. The accelerator structure here has azimuth symmetry. The proposed structure contains a diskloaded cylindrical waveguide where there is a periodical change of RF-field vs. azimuth. The modulation deforms the rf-field in such a manner that the accelerated particles undergo transverse focusing forces. The new class of accelerator structures covers the initial part of $\mathrm{e}+\mathrm{e}-$ linacs where a bunch is not rigid and additional transverse focusing fields are necessary. We discuss a bunch formation with a high transverse aspect ratio in the proposed structure and particularly in the photoinjector part of a linac.
\end{abstract}

\section{INTRODUCTION}

The proposed structure is a new type of e-/e+ accelerating structure, which combines a longitudinal acceleration with rf- transverse focusing/defocusing attributes. The working principle of the new accelerator structure is the same as the classical RFQ-structure that is widely used for the proton or heavy ion machines. The main difference is that the new structure runs with the electron/positron beams. The cross section of the first cell in the vertical plane is a typical cross section for a classical accelerator structure. The cross section in the horizontal plan for the same cell is different. If the bunch passes the structure from left to right, the first cavity wall has a slope for the horizontal plane. The slope of the iris wall is periodically changed as a function of azimuth $0<$ $\theta<2 \pi$. The left side of the iris surface wall has no azimuth variation and looks like a classical diskloaded waveguide surface. The inner surfaces in the second cell are the same as in the first cell except that the second cell is azimuthally rotated for $\theta=\pi / 2$. The slope of the vertical plane may be slightly different compared to the slope in the horizontal plane of the first cell. We are not discussing the common case when the following cell is azimuthally rotated for $\theta<\pi / 2$ (adiabatic rotation). The role of the azimuth wall modulation is a creation of the required potential distribution in the region where the bunch is propagated. To create the required potential distribution we propose employing a wall modulation that can be described (in polar coordinate system) in the next form

$$
\begin{aligned}
& x(\theta)=r \cdot \cos \theta \\
& y(\theta)=r \cdot \sin \theta \\
& z(\theta)=z_{0}+h \cdot|\sin \theta|^{n}
\end{aligned}
$$

where $\mathrm{a}<\mathrm{r}<\mathrm{b}$ are the cell radius, $\mathrm{z} 0$ is a local wall cell coordinate, $h$ is the depth of the wall modulation in the longitudinal direction, and $n$ is the degree of modulation (in practical case $n=2$ ). The depth and degree of modulation are cell parameters, which can create the required potential distribution in the cells. Fig. 1 shows one quarter of a model with two cells.

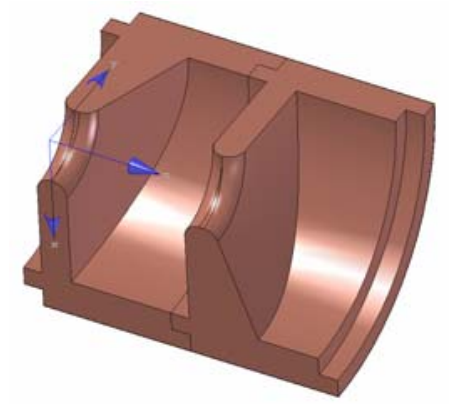

Fig. 1 Two cells of accelerating structure with cyclic variation of azimuth asymmetry (quarter model)

A level of modulation vs. azimuth is shown in Fig. 2. It has been shown that we can get the modulation of cell vacuum space as a function of $n$ amount.

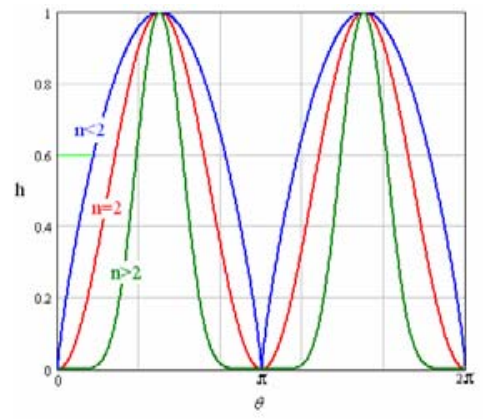

Fig. 2

Another way to get a spatial modulation of RF power in an acceleration structure is the employment of cyclic variation of elliptical coupling holes in the iris array. In classic accelerating structures, the coupling holes are round. To produce a spatial RF focusing effect we propose the accelerating structure that is shown in 
Fig. 3 (half of model with two cells is shown). Coupling holes are in elliptical form.

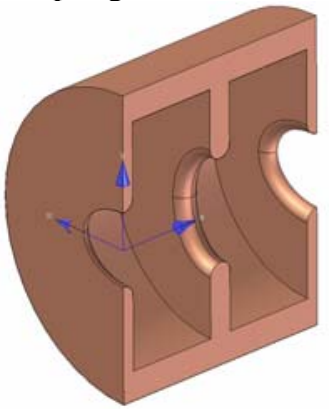

Fig. 3

The orientation of ellipse axis from hole-to-hole is shifted 90 degree. RF focusing field can be generated in such a structure too.

\section{EVALUATION OF FOCUSING STRENGTH}

A normalized transverse component of RFelectric field vs. normalized axial distance near the cell iris is shown in Fig. 4. A diagram shows the transverse field distribution for $1 \mathrm{~mm}$ axial offset and for $\mathrm{X}$ (red) and Y (blue) panes. Axial deviation is normalized on wavelength $\lambda$ and $z / \lambda=0$ corresponds to the axial center of iris.

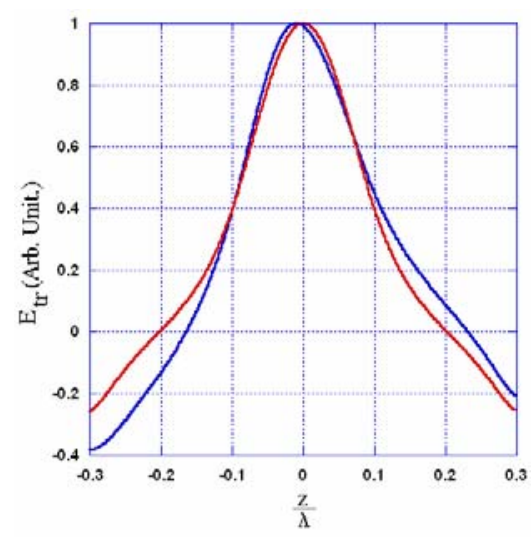

Fig. 4

A function Etr for the X-plane has a symmetry relative to $\mathrm{z} / \lambda=0$ and the focusing result is zero if the action of the space charge effect is negligible. The degree of asymmetry for the Y-plane (blue curve) is a figure of merit for the focusing effect. The evaluation of the ratio $\mathrm{R}$ between focusing and defocusing kicks for the S-band $\pi$-mode TM010 standing wave structure was made for the radial offset $1 \mathrm{~mm}$ and $5 \mathrm{~mm}$. The ratio $\mathrm{R}=1.43$ was obtained for the amplitude of a wall modulation $\mathrm{h}=$ $0.134 \lambda$ and $n=2$. It is clear that $R$ value can be different for different $\mathrm{h}, \mathrm{n}$, and other cell parameters. In order to get a clue how strong the amplitude alternating electric field is, we assume that the accelerating gradient in the structure is 100
$\mathrm{MeV} / \mathrm{m}$, then the evaluated focusing/defocusing amplitude Etr $\sim 260 \mathrm{kV} / \mathrm{cm}$ can be realized. MAFIA and HFSS 3D codes are used for evaluation of the focus/defocus effect in accelerating structures with a cyclic variation of azimuth asymmetry. The cell model and a pattern of the electrical field vector for $\mathrm{Y}$ - plane are illustrated in Fig. 5 after the HFSS simulation.

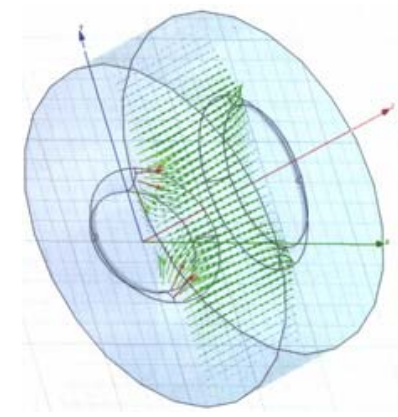

Fig. 5 Cell with elliptical coupling holes shifted on $90^{\circ}$

A stronger ratio can be realized for the structure with elliptical apertures. $\mathrm{R} \sim 2.2$ produces $\sim 400$ $\mathrm{kV} / \mathrm{cm}$ focusing gradient. The effective aperture radius was $12.5 \mathrm{~mm}$ in this case.

\section{EXAMPLE: WHERE PROPOSED STRUCTURES MAY BE EMPLOYED}

We would like to discuss implementation of the proposed structure for the photocathode gun and initial part of electro injector. The RF accelerating field increases with time when the bunch propagates through the cavity. The focusing RF force on the downstream iris will be weaker than on the upstream iris resulting in a transverse emittance growth. The new gun generates the flat bunch with the increased emittance aspect ratio. There are several methods that a flat bunch may be generated. In our case, the method of a flat bunch generation is based on a special spatial RF-field deformation near a photocathode in the first gun cell. Taking into account the conservation of the squared sum of beam emittance $\varepsilon_{n v}^{2}+\varepsilon_{n h}^{2}=i n v$ and the product of vertical and horizontal emittances $\varepsilon_{n v} \cdot \varepsilon_{n h}=i n v$, the proposed gun produces a lower emittance compared with the known photocathode round beam guns. The reduction of emittance is an actual problem for the LCLS, ILC, FELs, and other advanced accelerator based programs, in which a flat beam at the end is the preferable beam structure. A flat-bunch profile relieves the space-charge effects. A reduction of the space-charge effects is especially important in the earlier stages of acceleration and particularly in the area near a cathode where a relativism of particles is low. Space-charge effects control the 
transverse and longitudinal emittance growth. We offer photocathode gun geometry in which spacecharge effects will be reduced. We know it will be an ambitious goal to design a RF-gun that allows eliminating the damping ring in the ILC project. Should the accelerating structures with cyclic variation of azimuth asymmetry be implemented for this ambitious goal?

A cutoff in Y-pane of the proposed photocathode gun and one quarter of the first cavity wall with a photocathode cathode are shown in Fig. 6a and 6b accordingly. As you can see in Fig. 6b, there is a recessed wall with a cathode.

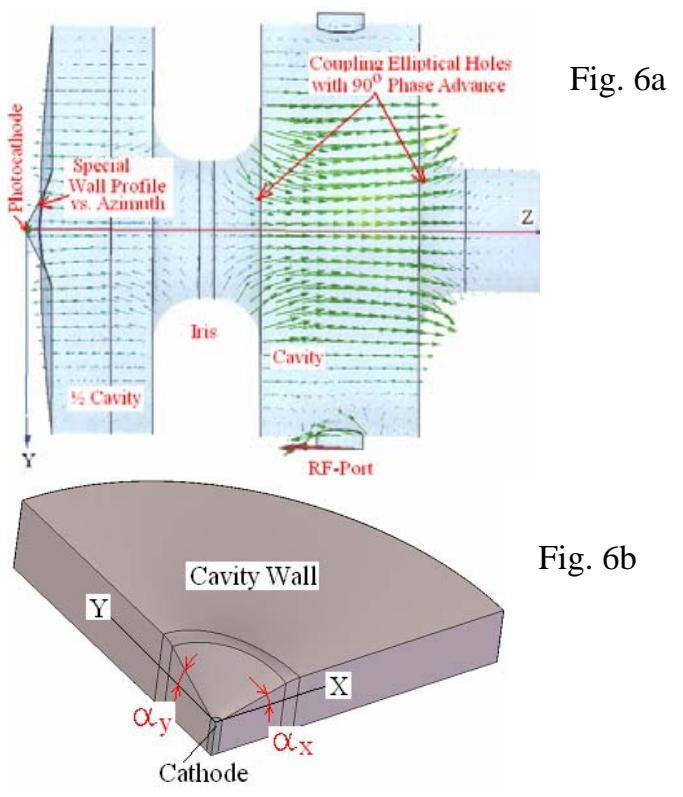

The recessed wall has an azimuth modulation, i.e. $\alpha_{x} \neq \alpha_{y}$. In case there is no recessed wall, the RF accelerating field increases with time when the bunch propagates through the cavity. The focusing $\mathrm{RF}$ force on the downstream iris will be weaker than on the upstream iris resulting in an emittance growth. The recessed wall will create the extra focusing force. In the earlier stages of acceleration, the longitudinal momentum of electrons is small. Electrons can easily be focused in one plane and symmetry of the initial round bunch may be broken. The transformation round-to-flat beam goes together with acceleration. The action of defocusing space-charge forces will be reduced in the earlier stages of the acceleration. To show how this idea works, a 3D beam simulation is required. The 3D MICHELLE code is employed to get the beam envelope through the gun. A fully relativistic equation is used for the beam dynamic (including the self magnetic field). The following results of simulations are for illustration only. The dimensions of the gun were taken close to the Sband mode operation.

Fig. 7a and 7b show the flat bunch formation. The aspect ratio of vertical-to horizontal planes is approximately 10 and the beam continues to compress vertically. However, the unstructured mesh in the beam envelope volume is rather coarse. There is a doubt that vertical size of the beam and its dynamic are simulated correctly. The reported result supports the qualitative analysis (proof of principal).

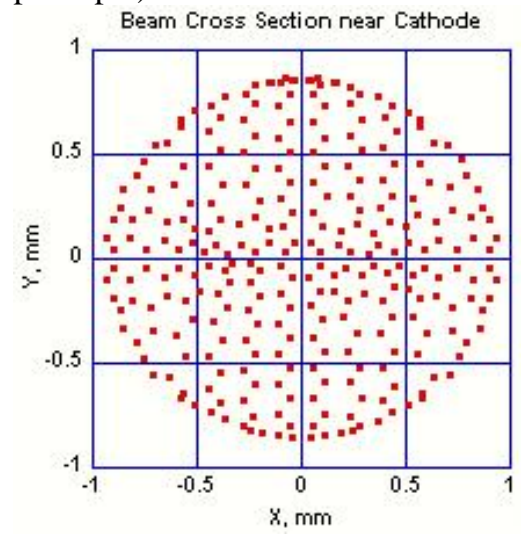

Fig. 7a Initial bunch cross section is round

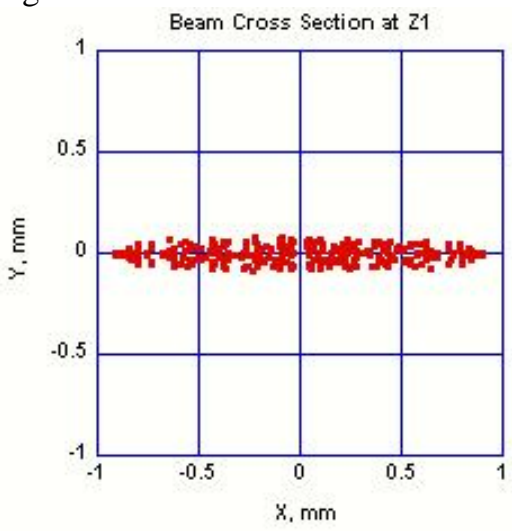

Fig. 7b Flat bunch is formed

\section{CONCLUSION}

A new class of accelerating structures is considered. A cyclic variation of azimuth asymmetry can produce the RF focus/defocus effect. The strength of effect depends on parameters of azimuthal variation. The proposed structures may be useful for the initial part of RF e+/e- linacs.

\section{ACKNOWLEDGMENT}

The author thanks the SLAC co-workers who were involved in useful discussion.

Work supported by the U.S. Department of Energy under contract number DE-AC0376 SF00515 